DOI: https://doi.org/10.15407/techned2021.04.003

\title{
EXACT ANALYTICAL AND APPROXIMATE ASYMPTOTIC CALCULATION METHODS TO DETERMINE THREE-DIMENSIONAL ELECTROMAGNETIC FIELD NEAR CONDUCTING BODY WITH FLAT SURFACE
}

\author{
Yu.M. Vasetsky* \\ Institute of Electrodynamics National Academy of Sciences of Ukraine, \\ pr. Peremohy, 56, Kyiv, 03057, Ukraine, e-mail: yuriy.vasetsky@gmail.com
}

The analytical solution of the three-dimensional quasi-stationary electromagnetic field problem for a current_located near conducting body with a flat surface is considered. The exact and approximate solution of the problem is presented. The exact solution has no restrictions on the external field configuration, physical properties of the medium, and frequency. The approximate solution is based on an expansion in asymptotic series and has limitations: for sinusoidal field, the solution is limited to frequencies above the lower limit; for pulsed field, the solution is limited by the initial time interval of the current pulse. Based on comparison of the results of exact and approximate calculations for nonuniform sinusoidal field at the interface between the media, the admissible value of the introduced small parameter is determined. For pulsed field the proposed choice of the limited time interval for calculating electomagnetic field using the asymptotic method is justified. References 29, figures 7.

Key words: three-dimensional electromagnetic field, exact analytical method, asymptotic series expansion, skin effect, sinusoidal and pulsed fields.

Introduction. Attention to the study of three-dimensional high-frequency sinusoidal and high-speed pulsed electromagnetic fields, taking into account eddy currents in conducting bodies is due to the need to take into account the influence of geometric and physical factors on the processes in the device elements of which a strong skin effect is appeared. It is enough to point out, for example, the technology of high-density pulsed currents to change the mechanical properties and control the stress-strain state of metal products [1-3], devices for high-speed forming technology using pulse magnetic field [4-6], equipment for high frequency induction heat treatment of metals $[7-10]$. Here, the search for the geometry of electromagnetic systems and the optimization of their parameters is of particular interest, which is associated with the significant laboriousness of computational procedures. These circumstances determine the need to develop of methods for electromagnetic field simulation, which provide high accuracy with a moderate amount of necessary calculations. Among them, analytical and numerical-analytical approaches stand out.

Considering that with strong skin effect, the current and the field are concentrated in a thin surface layer of the conductor, approximate calculation methods are often used to determine the electromagnetic field. The most advanced methods are those based on the perturbation method $[11,12]$. To determine the field in the dielectric region outside the conductor, an effective technique is to use the impedance boundary condition [13, 14], which establishes a connection between the components of the electric and magnetic fields tangential to the surface. The developed approaches are used in modeling electrodynamics problems taking into account the geometric and physical properties of real boundary surfaces $[15,16]$. A detailed analysis of the application of various numerical methods using the impedance boundary condition in electrodynamics problems is presented in the book [12].

A distinctive feature of this paper is the use of an exact analytical solution for a three-dimensional sinusoidal field of an arbitrary system of external sources located near the conducting half-space [17, 18]. The exact solution made it possible to obtain a number of general features of the electromagnetic field formation and to propose justified approximate methods for solving problems based on the asymptotic expansion of functions of the exact solution [19-21].

The found asymptotic approximation for the frequency spectrum of potentials and field vectors has limitations in the low-frequency range. However, the theoretical estimate of the calculation accuracy [22]

(C) Vasetsky Yu.M., 2021

ORCID ID: * https://orcid.org/0000-0002-4738-9872 
must be confirmed, at least by comparing the actual results obtained using exact and approximate expressions. In addition, it is advisable to show the validity of the values of the approximate expressions for the intensities of the nonuniform field at the interface between the media, which, as shown, are determined only by the values of the field of external sources.

Application of the asymptotic expansion for a pulsed electromagnetic field is based on a number of assumptions, which are associated with restrictions on the frequency spectrum. The validity of such assumptions must also be confirmed, including by comparing the calculation results of different methods.

The aim of the work is to substantiate theoretical estimation of the applicability range of the asymptotic method for calculating three-dimensional quasi-stationary electromagnetic field based on the comparison results of calculating the approximate and the exact analytical solutions for sinusoidal and pulsed fields.

1. Mathematical model. Exact analytical solution of the three-dimensional problem. The analytical solution of three-dimensional linear problem of conjugation of a quasi-stationary electromagnetic sinusoidal field at a plane interface between dielectric and conducting medium satisfies Maxwell's equations and boundary conditions. The boundary conditions include the equality of the normal components of the conduction density current in conducting medium and the displacement density current in dielectric medium.

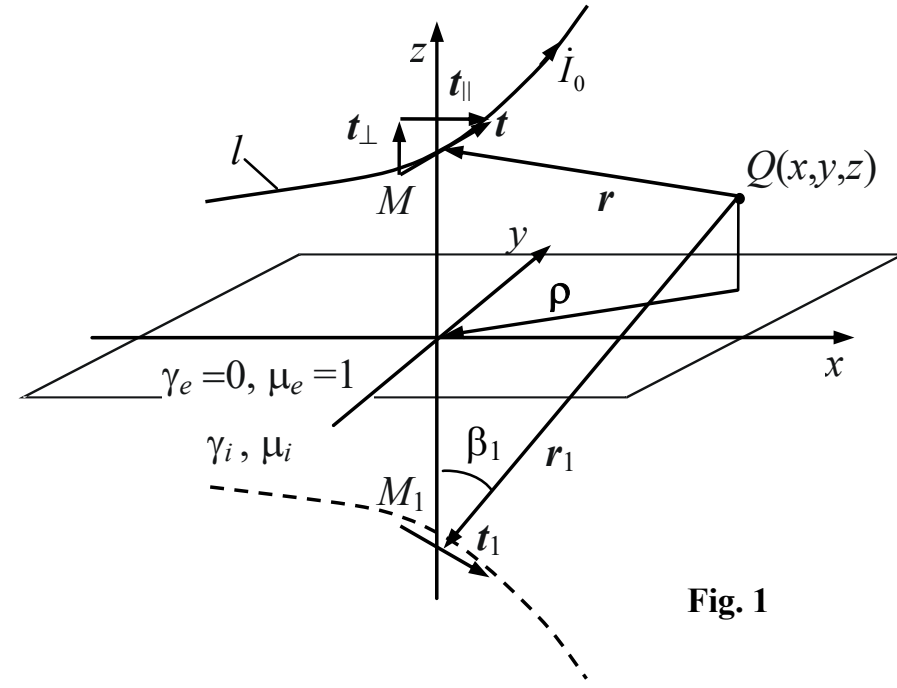
The solution is based on the known analytical solution of the problem for an emitting current dipole near the interface $[23,24]$. In the quasistationary approximation, a closed contour of arbitrary configuration located in a nonconducting and nonmagnetic medium, without loss of generality, was represented by a serial system of dipoles with a constant initial current $\dot{I}_{0}(i \omega)$ along the contour, where $\omega$ is cyclic frequency, $i$ is imaginary unit. The current contour is located in the dielectric medium near conducting half-space with electrical conductivity $\gamma$ and relative magnetic permeability $\mu$. The element of closed contour $l$ is shown in Fig. 1. The solution was obtained without restrictions of the contour configuration, properties of the medium and the frequency of the field. It easily extends to the general case of an arbitrary system of contours, that is, an arbitrary external field and to an arbitrary current dependence on time $I_{0}(t)$ using a Fourier transform.

1.1. Exact analytical solution for sinusoidal electromagnetic field. If external sources are represented by a single closed contour with current, then the expressions for the complex-value amplitudes of the vector $\dot{\boldsymbol{A}}$ and scalar $\dot{\varphi}$ potentials in the Lorentz gauge, the intensities of the electric $\dot{\boldsymbol{E}}$ and magnetic $\dot{\boldsymbol{H}}$ fields in the dielectric half-space can be represented in the form of the following contour integrals:

$$
\begin{gathered}
\dot{\boldsymbol{A}}_{e}=\frac{\mu_{0} \dot{I}_{0}}{4 \pi} \oint_{l}\left(\frac{\boldsymbol{t}}{r}-\frac{\boldsymbol{t}_{1}}{r_{1}}-\boldsymbol{t}_{1} \frac{\partial G}{\partial z}\right) d l, \\
\dot{\varphi}_{e}=i \omega \frac{\mu_{0} \dot{I}_{0}}{4 \pi} \oint_{l}\left(\boldsymbol{t}_{1} \cdot \boldsymbol{e}_{z}\right) G d l, \\
\dot{\boldsymbol{E}}_{e}=-i \omega \frac{\mu_{0} \dot{I}_{0}}{4 \pi} \oint_{l}\left(\frac{\boldsymbol{t}}{r}-\frac{\boldsymbol{t}_{1}}{r_{1}}-\boldsymbol{e}_{z} \times\left[\boldsymbol{t}_{1} \times \nabla G\right]\right) d l, \\
\dot{\boldsymbol{H}}_{e}=-\frac{\dot{I}_{0}}{4 \pi} \oint_{l}\left[\frac{\boldsymbol{t} \times \boldsymbol{r}}{r^{3}}-\frac{\boldsymbol{t}_{1} \times \boldsymbol{r}_{1}}{r_{1}^{3}}-\boldsymbol{t}_{1} \times \nabla\left(\frac{\partial G}{\partial z}\right)\right] d l .
\end{gathered}
$$

Here $\boldsymbol{t}$ and $\boldsymbol{t}_{1}$ are unit tangent vectors to the initial and mirror reflected from the surface contours at the source points $M$ and $M_{1}$; the position of these points relative to the observation point $Q$ is determined by vectors $\boldsymbol{r}$ and $\boldsymbol{r}_{1}$, respectively; the axis $z$ is oriented perpendicular to the interface surface in the direction 
of the unit vector $\boldsymbol{e}_{z}$. For arbitrary spatial contour, the unit tangent vector to the contour $\boldsymbol{t}=\boldsymbol{t}_{\|}+\boldsymbol{t}_{\perp}$ has nonzero projections onto the vertical direction $\boldsymbol{t}_{\perp}=\left(\boldsymbol{t} \cdot \boldsymbol{e}_{z}\right) \boldsymbol{e}_{z}$ and onto the interface between the media $\boldsymbol{t}_{\|}=\boldsymbol{t}-\left(\boldsymbol{t} \cdot \boldsymbol{e}_{z}\right) \boldsymbol{e}_{z}$.

Potentials and field intensities are determined by single function $G$

$$
G=\frac{2}{\sqrt{i}} \int_{0}^{\infty} \frac{\exp \left(-\chi \cos \beta_{1} / \varepsilon_{1}\right) J_{0}\left(\chi \sin \beta_{1} / \varepsilon_{1}\right)}{w_{1}(\chi)} d \chi,
$$

where the parameter $\varepsilon_{1}=\mu /\left(r_{1} \sqrt{\omega \mu \mu_{0} \gamma}\right)=\mu \delta /\left(\sqrt{2} r_{1}\right)$ is proportional to the ratio of the penetration depth of the uniform field $\delta=\sqrt{2 /\left(\omega \mu \mu_{0} \gamma\right)}$ to the distance $r_{1}, J_{0}($.$) is the Bessel function of the first kind of zero order, the$ denominator is $w_{1}(\chi)=\chi / \sqrt{i}+\sqrt{1+[\chi /(\mu \sqrt{i})]^{2}}$.

Expressions (1) - (5) completely determine the quasi-stationary electromagnetic field of the sinusoidal current of the arbitrary configuration contour, taking into account the eddy currents in the conducting half-space.

1.2. Exact analytical solution for pulsed electromagnetic field. Expressions (1) - (5) can be considered as the frequency spectrum of the potentials and vectors of the electromagnetic field, which is created in the dielectric half-space when current pulse $I_{0}(t)$ with frequency spectrum $\dot{I}_{0}(i \omega)$ flows along the contour. In this case, to obtain a solution in the form of time dependences, it is sufficient to perform the inverse Fourier transform. The corresponding expressions can be represented as follows:

$$
\begin{gathered}
\boldsymbol{A}(t)=\frac{\mu_{0}}{4 \pi} \oint\left[\left(\frac{\boldsymbol{t}}{r}-\frac{\boldsymbol{t}_{1}}{r_{1}}\right) I_{0}(t)-\boldsymbol{t}_{1} \frac{\partial V_{A}(t)}{\partial z}\right] d l, \\
\varphi(t)=\frac{\mu_{0}}{4 \pi} \oint_{l}\left(\boldsymbol{t}_{1} \cdot \boldsymbol{e}_{z}\right) V_{\varphi}(t) d l \\
\boldsymbol{E}(t)=-\frac{\mu_{0}}{4 \pi} \oint\left[\left(\frac{\boldsymbol{t}}{r}-\frac{\boldsymbol{t}_{1}}{r_{1}}\right) \frac{\partial I_{0}(t)}{\partial t}-\boldsymbol{e}_{z} \times\left[\boldsymbol{t}_{1} \times \nabla V_{\varphi}(t)\right]\right] d l, \\
\boldsymbol{H}(t)=-\frac{1}{4 \pi} \oint\left[\left(\frac{\boldsymbol{t} \times \boldsymbol{r}}{r^{3}}-\frac{\boldsymbol{t}_{1} \times \boldsymbol{r}_{1}}{r_{1}^{3}}\right) I_{0}(t)-\boldsymbol{t}_{1} \times \nabla\left(\frac{\partial V_{A}(t)}{\partial z}\right)\right] d l .
\end{gathered}
$$

The functions $V_{A}(t)$ and $V_{\varphi}(t)$ are written as inverse Fourier transform as following

$$
\begin{gathered}
V_{\mathrm{A}}(t)=\frac{2}{\pi} \int_{0}^{\infty} \cos (\omega t) \operatorname{Re}\left[\dot{I}_{0}(i \omega) G(i \omega)\right] d \omega, \\
V_{\varphi}(t)=\frac{2}{\pi} \int_{0}^{\infty} \cos (\omega t) \operatorname{Re}\left[i \omega \dot{I}_{0}(i \omega) G(i \omega)\right] d \omega .
\end{gathered}
$$

To describe the pulsed field, two functions $V_{A}(t)$ and $V_{\varphi}(t)$ are needed, and, however, each of them is still determined only by function $G(i \omega)$.

2. Asymptotic approximation. The above expressions are valid for any parameter values. At the same time, computationally, the determination of the potentials and vectors of the electromagnetic field, especially pulsed field, is associated with difficulties that are caused by the need to calculate improper triple integrals. Therefore, simplification of calculations is an important task. For this purpose, an approximate asymptotic method can be used, which is valid in the case of a strong skin effect [19,22].

2.1. Asymptotic approximation for frequency spectrum. To obtain approximate expressions, it is sufficient to use the expansion of the function $G$ in asymptotic series. The expansion is carried out according to a small parameter $\varepsilon_{1}<1$, which in this case reflects a significant degree of remoteness of the external field sources $r_{1}$ compared to the penetration depth $\delta$. It means that the asymptotic expansion is valid in the case of a strong skin effect, in which the penetration depth of the field is small not only with respect to the characteristic dimensions of conducting body, but also of the entire electromagnetic system, including the distance from the surface of body to the external sources. The small parameter value determines the 
limitation on the field frequency. The frequency $f=\omega / 2 \pi$ must be large than the limit value $f>f_{m}=\mu /\left(2 \pi \mu_{0} \gamma r_{1}^{2} \varepsilon_{m}^{2}\right)$, where $\varepsilon_{m}$ is the chosen permissible value of the small parameter.

A feature of asymptotic series is that they are divergent series. In this case, the function $G$ is expanded into asymptotic power series of the Poincaré type $[25,26]$ with an error that can be made arbitrarily small by choosing $\varepsilon_{1} \rightarrow 0$. With an increase in the number of terms in the series, the error in the approximation of the function first decreases, reaching a minimum, which depends on the value of the small parameter. Then the approximation error increases. In addition, each term of the asymptotic series is determined with an error, the magnitude of which depends on the value of the small parameter and the number $n$ of the term in the series. Therefore, for each term of the series, there is also a limitation on the lower value of the frequency $f \geq f_{n}$, which increases with increasing number of the member of the series $n$. In this regard, with an increase in the value of the small parameter, the total number of considered members of the series $N$ decreases and, accordingly, the total approximation error increases.

The asymptotic series for the function $G$ takes the following form [22]:

$$
\dot{G} \approx \sum_{n=0}^{N} \dot{G}_{n}=\sum_{n=0}^{N} 2(-1)^{n} a_{n}(\mu)\left(\frac{\varepsilon_{1}}{\sqrt{i}}\right)^{n+1} r_{1}^{n+1} \frac{\partial^{(n)}}{\partial z^{n}}\left(\frac{1}{r_{1}}\right),
$$

where $a_{n}(\mu)$ are the Taylor series coefficients of the function $1 / w_{1}=\sum_{n=0}^{\infty} a_{n}(\mu)(\chi / \sqrt{i})^{n}$.

Taking into account (12), expressions (1) - (4) can be written as follows:

$$
\begin{aligned}
& \dot{\boldsymbol{A}}=\frac{\mu_{0} \dot{I}_{0}}{4 \pi}\left[\oint_{l}\left(\frac{\boldsymbol{t}}{r}-\frac{\boldsymbol{t}_{1}}{r_{1}}\right) d l-\sum_{n=0}^{N}(-1)^{n} 2 a_{n}(\mu)\left(\frac{\mu}{p}\right)^{n+1} \frac{\partial^{(n+1)}}{\partial z^{n+1}} \oint_{l} \frac{\boldsymbol{t}_{1}}{r_{1}} d l\right], \\
& \dot{\varphi}=-\frac{\dot{I}_{0}}{4 \pi} \varsigma \sum_{n=0}^{N}(-1)^{n} 2 a_{n}(\mu)\left(\frac{\mu}{p}\right)^{n} \frac{\partial^{(n)}}{\partial z^{n}} \oint_{l} \frac{\left(\boldsymbol{t}_{1} \cdot \boldsymbol{e}_{z}\right)}{r_{1}} d l, \\
& \dot{\boldsymbol{E}}=-i \omega \frac{\mu_{0} \dot{I}_{0}}{4 \pi} \oint_{l}\left(\frac{\boldsymbol{t}}{r}-\frac{\boldsymbol{t}_{1}}{r_{1}}\right) d l+\frac{\dot{I}_{0}}{4 \pi} \zeta \sum_{n=0}^{N}(-1)^{n} 2 a_{n}(\mu)\left(\frac{\mu}{p}\right)^{n} \frac{\partial^{(n)}}{\partial z^{n}} \boldsymbol{e}_{z} \times \oint_{l} \frac{\boldsymbol{t}_{1} \times \boldsymbol{r}_{1}}{r_{1}^{3}} d l . \\
& \dot{\boldsymbol{H}}=-\frac{\dot{I}_{0}}{4 \pi}\left[\oint_{l}\left(\frac{\boldsymbol{t} \times \boldsymbol{r}}{r^{3}}-\frac{\boldsymbol{t}_{1} \times \boldsymbol{r}_{1}}{r_{1}^{3}}\right) d l-\sum_{n=0}^{N}(-1)^{n} 2 a_{n}(\mu)\left(\frac{\mu}{p}\right)^{n+1} \frac{\partial^{(n+1)}}{\partial z^{n+1}} \oint_{l}^{\boldsymbol{t}_{1} \times \boldsymbol{r}_{1}} \frac{r_{1}^{3}}{r^{3}} d l\right] .
\end{aligned}
$$

Here, it is taken into account that $\varepsilon_{1} r_{1} / \sqrt{i}=\mu / p$, where $p=\sqrt{i \omega \mu_{i} \mu_{0} \gamma_{i}}$ is propagation constant, $\varsigma=p / \gamma_{i}$ is surface impedance.

In the case of strong skin effect, the distribution of the electromagnetic field at the interface between the media is of great importance. The electromagnetic field on this surface defines such characteristics as the energy flow of the electromagnetic field into the conductive body, the surface density of Joule heat release in the surface layer, the magnetic pressure on the surface of the body. Expressions (15) and (16) for the intensities of the electromagnetic field at $z=0$ are greatly simplified. Here at strong skin effect the electromagnetic field is determined only by the known distribution of the field of external sources at the boundary without the need to solve additional equations [20,22]:

$$
\begin{aligned}
& \dot{\boldsymbol{E}}_{\|}(z=0)=\sum_{n=0}^{N} \dot{\boldsymbol{E}}_{\| n}=\left.\varsigma \sum_{n=0}^{N} 2 a_{n}(\mu)\left(\frac{\mu}{p}\right)^{n}\left\{\frac{\partial^{(n)}}{\partial z^{n}} \boldsymbol{e}_{z} \times \dot{\boldsymbol{H}}_{0 \|}\right)\right|_{z=0}, \\
& \dot{\boldsymbol{H}}_{\|}(z=0)=\sum_{n=0}^{N+1} \dot{\boldsymbol{H}}_{\| n}=-\left.\sum_{n=0}^{N+1} 2 a_{n-1}(\mu)\left(\frac{\mu}{p}\right)^{n}\left\{\frac{\partial^{(n)} \dot{\boldsymbol{H}}_{0 \|}}{\partial z^{n}}\right\}\right|_{z=0}, \\
& \dot{\boldsymbol{H}}_{i \perp}(z=0)=\sum_{n=0}^{N} \dot{\boldsymbol{H}}_{i \perp n}=\left.\sum_{n=0}^{N} 2 \frac{a_{n}(\mu)}{\mu}\left(\frac{\mu}{p}\right)^{n+1}\left\{\frac{\partial^{(n+1)} \dot{\boldsymbol{H}}_{0 \perp}}{\partial z^{n+1}}\right\}\right|_{z=0},
\end{aligned}
$$


where $\dot{\boldsymbol{H}}_{0}$ is the magnetic field intensity of external sources in dielectric medium at the interface; $\dot{\boldsymbol{H}}_{0 \|}$ and $\dot{\boldsymbol{H}}_{0 \perp}$ are the tangent and normal components of the field, respectively; it is accepted $a_{-1}=-1$. If the external field is created by a single current contour, then

$$
\dot{\boldsymbol{H}}_{0}=-\frac{\dot{I}_{0}}{4 \pi} \oint \frac{\boldsymbol{t} \times \boldsymbol{r}}{r^{3}} d l .
$$

2.2. Asymptotic approximation for pulsed field. To find pulsed electromagnetic field in the dielectric half-space, it is necessary to determine the functions $V_{A n}(t)$ and $V_{\varphi n}(t)$ for given time dependence of the current $I_{0}(t)$. The values $V_{A n}(t)$ and $V_{\varphi n}(t)$ are determined by expressions similar to (10) and (11), with replace $G(i \omega)$ by $G_{n}(i \omega)$.

It is essential that each term of the asymptotic series is represented as a product of two functions, one of which depends on the frequency, and the other depends on the properties of the medium and the coordinates of the vector $\boldsymbol{r}_{1}$

$$
G(i \omega) \approx \sum_{n=0}^{N} G_{n}(i \omega)=\sum_{n=0}^{N} \frac{g_{n}\left(\mu, \gamma, \boldsymbol{r}_{1}\right)}{(i \omega)^{(n+1) / 2}} .
$$

Here the functions $g_{n}$ are as follows

$$
g_{n}=g_{n}\left(\mu, \gamma, \boldsymbol{r}_{1}\right)=(-1)^{n} 2 a_{n}(\mu)\left(\frac{\mu}{\sqrt{\mu_{0} \mu \gamma}}\right)^{n+1} \frac{\partial^{(n)}}{\partial z^{n}}\left(\frac{1}{r_{1}}\right)
$$

Taking into account the simple frequency dependence of each term in series (21), we will solve the problem in two stages. First, we find the time dependence under the action of unit current pulse $T(t)$

$$
T(t)= \begin{cases}0, & t<0 \\ 1, & t \geq 0\end{cases}
$$

At the next stage, using the Duhamel integral, we will find functions $V_{A n}(t)$ and $V_{\varphi n}(t)$ for an arbitrary dependence of the current on time $I_{0}(t)$.

Taking into account that the integrand (21) in the improper integral of the inverse Fourier transform does not satisfy the boundedness condition, we will use the Laplace operator $s$ instead of the operator $i \omega$.

Under the action of unit current pulse, taking into account (21), for each term of the asymptotic expansion $\Lambda_{A n}(s)=T(s) G_{n}(s)$ and $\Lambda_{\varphi n}(s)=s T(s) G_{n}(s)$ the inverse Laplace transform is a power function of time [27] $\lambda_{A n}(t)=t^{(n+1) / 2} g_{n} / \Gamma((n+3) / 2)$ and $\lambda_{\varphi n}(t)=t^{(n-1) / 2} g_{n} / \Gamma((n+1) / 2)$, where $\Gamma(\cdot)$ is the gamma function.

Since for all $\lambda_{A n}(0)=0$, then for the function $V_{A}(t)$ which determines the vector potential and magnetic field intensity, we apply the Duhamel integral in the following form [28]

$$
V_{A}(t)=\lambda_{A}(0) I_{0}(t)+\int_{0}^{t}\left[\left.\frac{d \lambda_{A}(\xi)}{d \xi}\right|_{\xi=t-\tau} I_{0}(\tau)\right] d \tau=\sum_{n=0}^{N} \frac{n+1}{2 \Gamma((n+3) / 2)} g_{n} \int_{0}^{t}(t-\tau)^{(n-1) / 2} I_{0}(\tau) d \tau .
$$

When calculating the scalar potential and electric field intensity, it is necessary to take into account that at $t \rightarrow 0$ the zero term of the series $\lambda_{\varphi 0}(t) \sim t^{-1 / 2}$ increases indefinitely. Therefore, to eliminate the singularity of the integrand, we will apply the Duhamel integral form, in which time derivative of current is used

$$
V_{\varphi}(t)=\lambda_{\varphi}(t) I_{0}(0)+\int_{0}^{t}\left[\left.\frac{d I_{0}(\xi)}{d \xi}\right|_{\xi=t-\tau} \lambda_{\varphi}(\tau)\right] d \tau=\sum_{n=0}^{N} \frac{g_{n}}{2 \Gamma((n+1) / 2)} \int_{0}^{t}\left[\left.\frac{d I_{0}(\xi)}{d \xi}\right|_{\xi=t-\tau} \tau^{(n-1) / 2}\right] d \tau .
$$

Here, it is necessary to impose a limitation on the dependence of the current on time near $t=0$. If the current changes according to the power law $I_{0}(t) \approx a t^{k}$, it is necessary $k>1 / 2$. An even stricter restriction is associated with the first two terms in (8), for which it is necessary $k \geq 1$. 
In the asymptotic approximation, the limitation on the field frequency makes it necessary to limit the frequency spectrum of the pulsed field in the low-frequency range. For current pulse this is due to limitation of the time interval over which the field can be calculated. For example, when a unit current pulse flows along the contour, the presence of low frequencies in the spectrum leads to an unlimited growth of functions $\lambda_{A n}(t)$ at $t \rightarrow \infty$, while the vector potential and magnetic field intensity should tend to a constant value. The expressions will be valid until a certain characteristic point in time $t_{c} \approx 1 / f_{c}$, for which, in fact, the previous condition is satisfied $\varepsilon_{1}=\mu \sqrt{t_{c}} /\left(2 \pi r_{1} \sqrt{\mu_{0} \mu \gamma}\right)<1$. The assessment of the allowable time intervals can be performed as follows: for the entire calculation $t \leq t_{m}=1 / f_{m}$ and for each term of the series $t \leq t_{n}=1 / f_{n}$. In the results below, the current is normalized to the maximum value $I_{0}^{*}(t)=I_{0}(t) / I_{0 \max }$.

\section{Comparison of exact and approximate calculation.}

3.1. Comparison of calculation results for sinusoidal field. The value of the small parameter $\varepsilon_{1}$ depends on the distance $r_{1}$ and, accordingly, changes depending on the relative position of the point $M_{1}$ on the mirrored contour and the observation point. For specific contour the parameter $\varepsilon_{1}$ takes its greatest value at the smallest distance $r_{1}$, when the observation point is located at the interface between the media on vertical axis passing through the point on the contour. Therefore, the largest error will occur when the field is calculated at the interface between media. In this regard, we compare the results of calculations using exact and approximate expressions for the field on the surface of the conducting half-space.

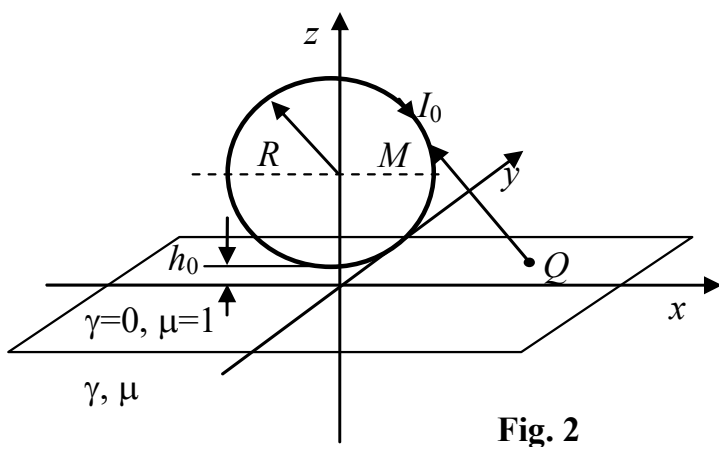

The analysis of the calculation errors of the sinusoidal field depending on the value of the parameter $\varepsilon_{1}$ for specific points $M_{1}$ not related to the configuration of the contour was studied in sufficient detail in [22]. Therefore, here we will compare the calculation of threedimensional electromagnetic field for a model of an electromagnetic system with circular contour with sinusoidal current [3] (Fig. 2). The contour lies in a plane normal to the interface between the media. The geometric dimensions are as follows: radius of the contour $R=0.05 \mathrm{~m}$, distance from the center of the contour to the

surface $H=0.06 \mathrm{~m}$, respectively, the minimum distance from the contour to the surface $h_{0}=0.01 \mathrm{~m}$. The electrophysical properties of the medium correspond to those of aluminum $\gamma=3,71 /(\Omega \cdot m), \mu=1$. Frequency is variable.

The calculation was carried out for all components of the electric and magnetic field intensities according to exact (3) - (5) and approximate (17) - (20) expressions. The results are presented for the normalized component values of the complex-value amplitudes of the electric $\dot{E}_{k}^{*}= \pm\left|E_{k}^{*}\right| \exp \left(i \varphi_{E k}\right)$ and magnetic $\dot{H}_{k}^{*}= \pm\left|H_{k}^{*}\right| \exp \left(i \varphi_{H k}\right)$ fields, where $k=x, y, z$. The argument of the complex-value amplitude shows the phase shift angle relative to the phase of the contour current within the limits $-\pi / 2 \leq \varphi \leq \pi / 2$ (the "-" sign in front of the complex-value amplitude modulus is equivalent to phase change by $\pi$ ). The normalized values of the field vectors are defined as follows $\dot{\boldsymbol{E}}=\frac{\mu_{0}\left|\dot{I}_{0}\right| \omega}{4 \pi} \dot{\boldsymbol{E}}^{*}, \dot{\boldsymbol{H}}=\frac{\left|\dot{I}_{0}\right|}{4 \pi h_{0}} \dot{\boldsymbol{H}}^{*}$.

In Fig. 3 for the point on the surface $x=0, y=0$ closest to the contour, nonzero normalized values of the components of the electric $\dot{E}_{x}^{*}$ and magnetic $\dot{H}_{y}^{*}$ field intensities are presented depending on the value of the parameter $\varepsilon=\mu /\left(r_{1 \min } \sqrt{2 \pi f \mu \mu_{0} \gamma}\right)$, where in this case the minimum distance of all $r_{1}$ is $r_{1 \min }=h_{0}$. Modules of complex-value amplitudes for calculation by exact (solid curves) and approximate (dashed curves) expressions are shown in Fig. $3 a, c$. The arguments for the complex-value amplitudes are given in Fig. $3 b, d$. 

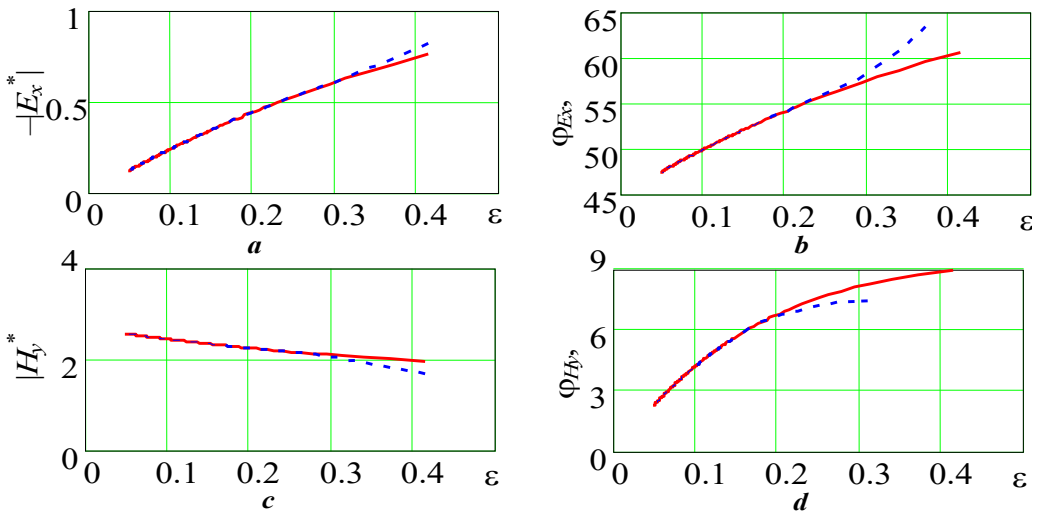

Fig. 3
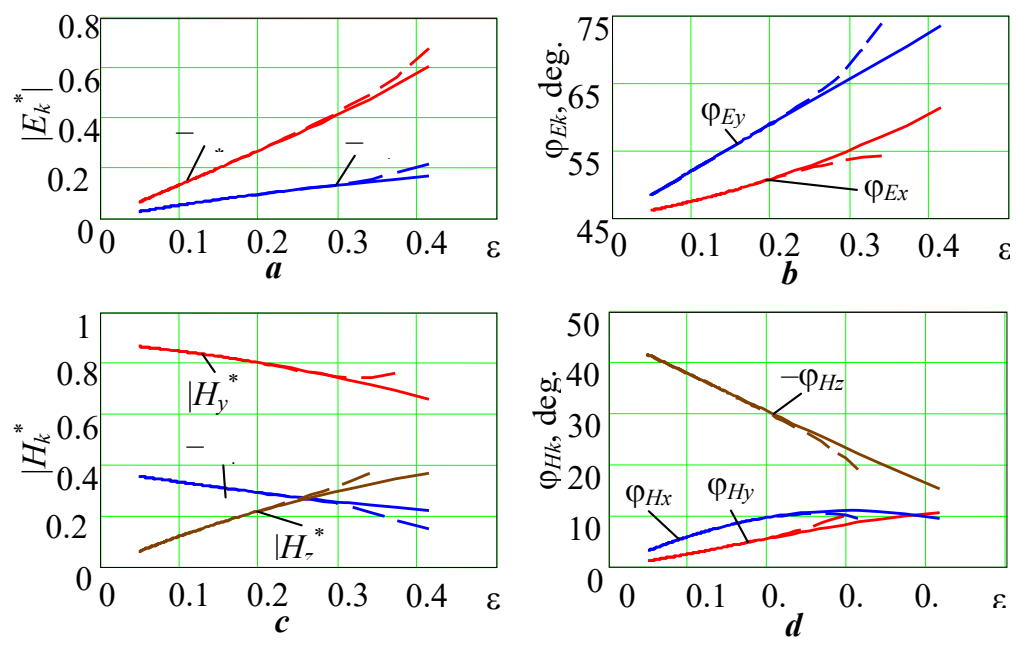

Fig. 4

only by the induced electrical field of external sources [17].

From the point of view of the possibility of using computationally simpler asymptotic expansion, the main conclusion is that for all components of the electromagnetic field, the results practically coincide with the calculation using exact expressions up to the value of a small parameter $\varepsilon_{m} \approx 0.3$. In addition, it is essential that the introduced small parameter, which combines several quantities, is a single parameter that indicates the limiting value for the application of approximate asymptotic calculation method. So, for the considered point on the surface, which is at a greater distance from the contour $r_{1}=0.018 \mathrm{~m}>h_{0}=0.01 \mathrm{~m}$, the limiting value of the field frequency decreases to the value $f_{m}=11.7 \mathrm{~Hz}$.

3.2. Comparison of calculation results for pulsed electromagnetic field. Since in the asymptotic expansion method the lower frequency limits increase with an increase in the number of term in the series, then in the initial period the largest number of the terms in the series is taken into account and the field can be calculated most accurately. The validity of the proposed estimate of the time intervals for the integrands in the contour integrals is carried out on the basis of comparing the calculation using exact expressions (5) (7) for functions $V_{A}(t), V_{\varphi}(t)$ and approximate expressions (24), (25). In addition, we will compare the results of calculating the electromagnetic field created by specific contours with pulsed current.

It is convenient to analyze expressions using dimensionless parameters. The basic frequency $f_{b}=\left(\pi h^{2} \mu \mu_{0} \gamma\right)^{-1}$ (and accordingly the time $t_{b}=1 / f_{b}$ ) is determined as the frequency when the penetration depth is equal to the vertical coordinate $\delta=h$ of a contour element. In this case, the normalized time is related to the value of the small parameter as $t^{*}=t f_{b}=2\left(\varepsilon_{1} / \mu\right)^{2}\left(r_{1} / h\right)^{2}$. The contour current in the results below is normalized to the maximum value $I_{0}^{*}(t)=I_{0}(t) / I_{0 \max }$. 
Fig. 5 illustrates a comparison of the exact and approximate normalized values of the function $V_{A}^{*}(t)=V_{A}(t) / I_{0 \max }$ for an exponentially decaying current pulse, which at the initial moment $t=0$ takes on a maximum value by jump. At the point on the surface under the contour element (Fig. $5 a$ ) at $\rho=0, r_{1}=h$ and $\mu=1$ for the chosen permissible value of the small parameter, for example, $\varepsilon_{m}=0.3$ the limit value of the considered time interval turns out to be $t_{m}^{*}=0.18$. The insignificant deviation of the calculation results at $t^{*} \sim 0.12$ practically disappears when the distance $r_{1}=\sqrt{h^{2}+\rho^{2}}$ increases (Fig. $5 b$ ). Here, for the same value of small parameter $\varepsilon_{m}=0.3$, the time intervals taken into account turn out to be much larger: $\rho / h=1, t_{m}=0.36$ and $\rho / h=3, t_{m}=1.8$. Deviations between the results of exact and approximate calculations within the allowable time intervals are insignificant and are not reflected in the graphs. It follows that, when integrating along the contour using the asymptotic approximation, the calculation error is mainly influenced by the calculation of the integrand at the source point closest to the observation point.

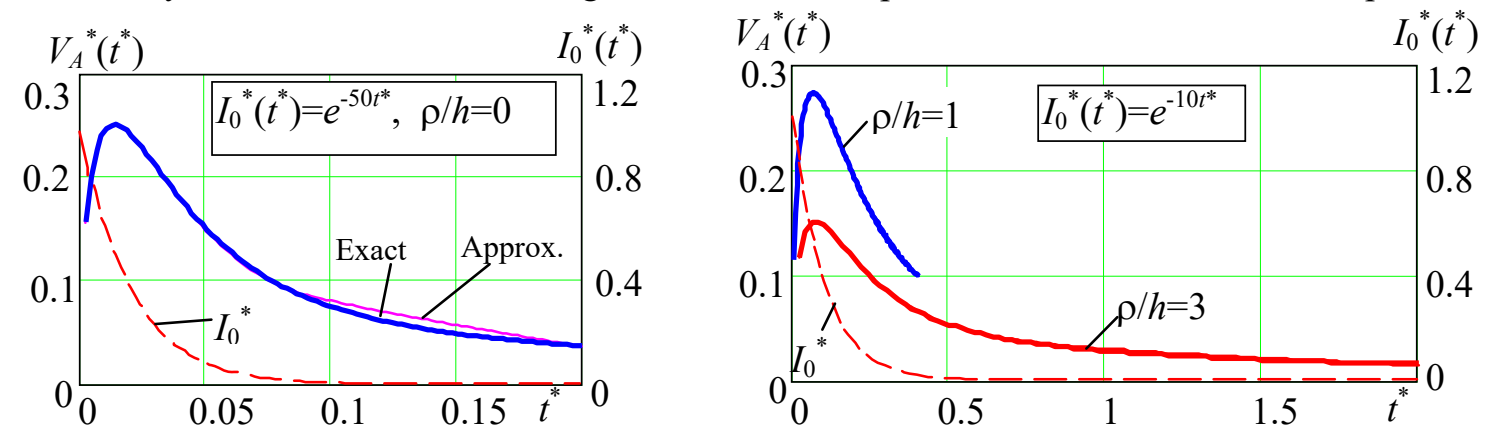

Fig. 5

Unlike the function $V_{A}(t)$ for calculating the function $V_{\varphi}(t)$, which determines the time dependence of the scalar potential and the electric field intensity, the current pulse should not change by jump. Otherwise, the field intensity will take on infinite value. G. Knopfel [31] also points out incorrect physical consequences when using models with a jump in the external field at the initial moment of time. For this reason, current pulse was chosen in the form of the difference of two exponentially decaying functions $I^{*}\left(t^{*}\right)=I_{m}^{*}\left(e^{-\alpha_{1}^{*} t^{*}}-e^{-\alpha_{2}^{*} t^{*}}\right)$, which at $t^{*} \rightarrow 0$ changes proportionally to time $t^{*} \rightarrow 0: I^{*}\left(t^{*}\right)=I_{m}^{*}\left(\alpha_{2}^{*}-\alpha_{1}^{*}\right) t^{*}$.

The results of comparing exact and approximate calculations of the normalized values of the function $V_{\varphi}^{*}\left(t^{*}\right)=V_{\varphi}(t) /\left(I_{0 \max } f_{b}\right)$ at a point on the surface $z=0, \rho=0$ for some current pulses are shown in Fig. 6 . It is seen that the approximate method of asymptotic expansion gives very insignificant deviations in comparison with the exact one in the time interval from the beginning of the current pulse action to the limiting value $t_{m}^{*}=0.18$. For the pulses in Fig. $6 b, c$ the values practically coincide over the entire time interval.
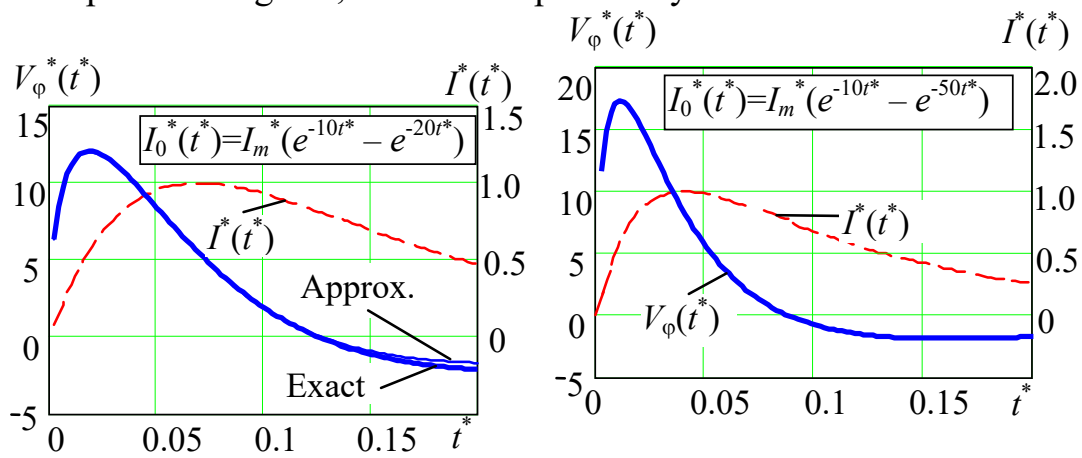

Fig. 6

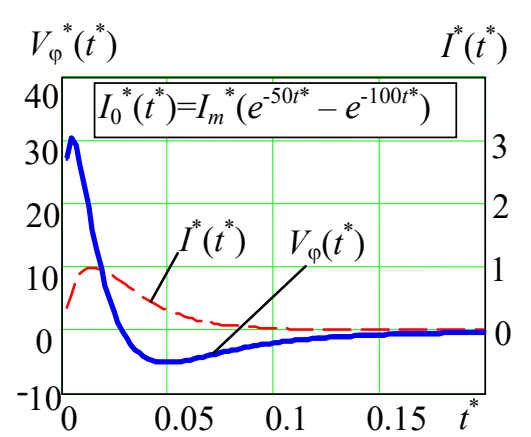

Since the small parameter $\varepsilon_{1}$ is function of points on the contour, the permissible calculation time interval also changes when integrating along the contour. For the entire contour, it is advisable to choose the minimum value of the permissible time interval, which corresponds to the minimum distance between the mirrored contour and the observation point. In this case, the accuracy of the field calculating for the entire contour will be higher than for the contour point with the greatest $\varepsilon_{1}$. 
The noted circumstance in Fig. 7 is illustrated by the time dependences of the vector potential and the electric field intensity for pulsed current. The results for the vector potential when pulsed current $I_{0}^{*}\left(t^{*}\right)=\exp \left(-50 t^{*}\right)$ flows along an elliptical contour are presented. The contour geometry and orientation relative to the boundary surface are given by the following parametric equations:

$$
x=a \cos \theta \cos \alpha, y=b \sin \theta, z=H-a \cos \theta \sin \alpha,
$$

where the parameter $\theta$ changes within $0 \div 2 \pi ; \alpha$ is the tilt angle of the plane in which the contour lies relative to the interface between the media; $H$ is the height of the location of the ellipse center relative to the boundary surface. The relative sizes of the semiaxes of the ellipse and tilt angle are chosen $a / H=1, b / H=2$ and $\alpha=60^{\circ}$. The contour is shown in Fig. $7 a$ on the left. The vector potential is determined at the point $Q(x / H=0,5 ; 0 ; 0)$ marked in the Figure with a cross, where the distance from the contour to the interface is minimal $r_{1 \min }^{*}=0.134$. At this point, for the chosen geometry of the contour, the vector potential has only a tangent component, parallel to the axis $y$.

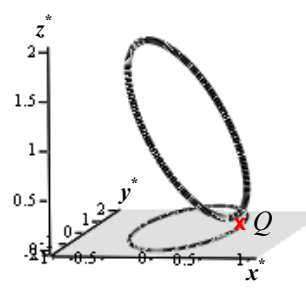

$a$

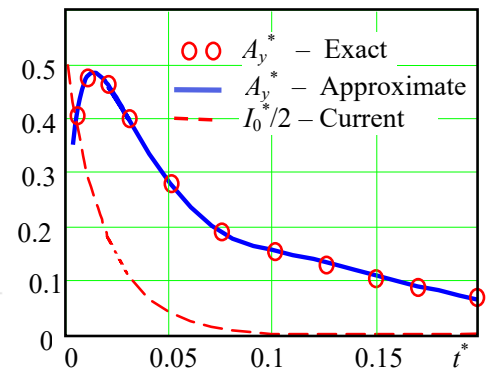

$15 t$

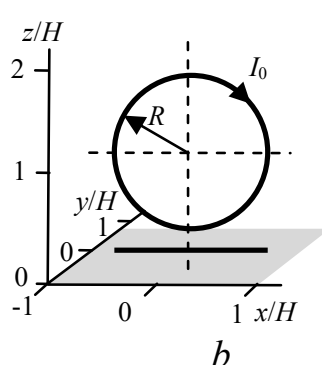

$b$

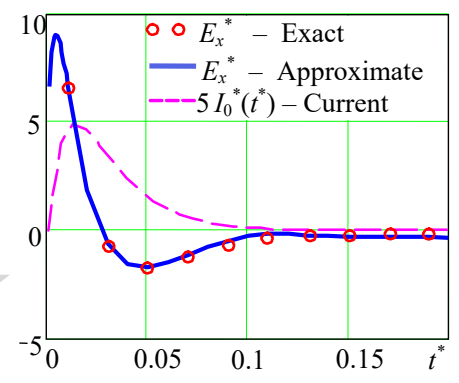

Fig. 7

The calculation results obtained by approximate expressions (13) are shown in Fig. 7, $a$ with a solid curve. Individual points show the results of calculations using exact analytical expressions (1), (5). The normalized values of the vector potential are determined in accordance with the expression $A_{Q}=\frac{\mu_{0} I_{m}}{4 \pi} A_{Q}^{*}$. It can be seen from the given dependences that the values of the vector potential calculated by the approximate expression coincide with the calculation by the exact expressions from the beginning of the pulse action to its normalized value $t^{*} \approx 0,2$. At this time interval, the calculation accuracy is higher than for the function $V_{A}^{*}\left(t^{*}\right)$ at the point of the contour located closest to the interface between the media (Fig. 5, $a$ ).

The electric field intensity of the circular contour (Fig. 7, b) with pulsed current $I_{0}^{*}\left(t^{*}\right)=4\left[\exp \left(-50 t^{*}\right)-\exp \left(-100 t^{*}\right)\right]$ was determined at the interface between the media at the point closest to the contour. The contour, as before, is located in a plane perpendicular to the boundary surface. The ratio of the circle radius $R$ to the distance $H$ from its center to the surface is $R / H=0.833$. In this case, the minimum distance of the contour from the surface is equal to $r_{1 \min }^{*}=0.167$. It can be seen that for the selected current pulse, the induced electric field decays before the maximum time value $t_{m}^{*}=0,18$ is reached, and throughout the entire transient process, the exact and approximate values practically coincide.

Comparison of the results of calculating the pulsed electromagnetic field by approximate and exact expressions confirms the validity of the choice of time intervals during which the use of approximate asymptotic method is permissible.

Conclusion. The presented analytical expressions for calculating the potentials and intensities of pulsed electromagnetic field are based on the exact analytical solution of the three-dimensional quasistationary problem for calculating of the field in the system "current contour - conductive half-space". There are no restrictions on the geometry of the contour, the physical properties of the medium and the frequency, and hence the dependence of the pulse current and the field on time.

In the case of a strong skin effect, the presented approximate expressions for nonuniform sinusoidal electromagnetic field make it possible, without solving additional equations, to find the electric and magnetic field intensities at the interface between the media, having only the known field distribution of external sources at the interface. Comparison of the results of exact and approximate field calculations made it 
possible to establish the limit value of the introduced small parameter, up to which the asymptotic expansion of expressions for the field at the interface is valid.

Since for pulsed field the exact analytical solution in the frequency domain involves the inverse Fourier transform and its obtaining is associated with calculating triple improper integrals, then under the strong skin effect it is reasonable to use the asymptotic expansion method for pulsed processes. It is shown that the calculation of the vector potential and the magnetic field strength can be performed for discontinuous time dependency of current. On the contrary, in calculating the scalar potential and electric field intensity, the finite rise time of the external field must be taken into account. Due to in the method of asymptotic expansion the values of the lower frequencies cutoff increase with increasing number of the series term, the field in the initial period can be calculated most accurately. Since the current pulse usually changes most rapidly and reaches its maximum values over a relatively short period of time, so during this, most important stage, the electromagnetic field is determined.

Comparison of the results of calculating the pulsed field by exact and approximate methods shows a high accuracy of calculations using the asymptotic expansion within the proposed time interval. Taking into account a significant reduction in the amount of calculations, the approximate method is effective method for calculating the distribution of the three-dimensional field.

Роботу виконано за бюджетною темою «Розробити нові моделі та методи дослідження електродинамічних процесів в електроенергетичному устаткуванні (генератори, трансформатори, двигуни власних потреб та ін.) для вирішення задач підвищення його надійності, контролю $і$ діагностики» (шифр «КОМПЛЕКС-4»), КПКВК 6541030.

1. Babutsky A., Chrysanthou A., Ioannou J. Effect of pulsed electric current treatment on corrosion of structural metals. Strength of materials. 2009. Vol. 41. No 4. Pp. 387-391. DOI: https://doi.org/10.1007/s11223-009-9142-3.

2. Gallo F., Satapathy S., Ravi-Chandar K. Melting and crack growth in electrical conductors subjected to shortduration current pulses. International Journal of Fracture. 2011. Vol. 16. Pp. 183-193. DOI: https://doi.org/10.1007/s10704-010-9543-0.

3. Vasetsky Yu.M., Kondratenko I.P. Electromagnetic field of the inductor for local electric pulse effects on metal products. Tekhnichna Elektrodynamika. 2020. No 4. Pp. 11-14. DOI: https://doi.org/10.15407/techned2020.04.011. (Ukr).

4. Psyk V., Risch D., Kinsey B.L., Tekkaya A.E., Kleiner M. Electromagnetic forming-A review. Journal of Materials Processing Technology. 2011. Vol. 211. Issue 5. Pp. 787-829. DOI: https://doi.org/10.1016/j.jmatprotec.2010.12.012.

5. Gayakwada D., Dargara M. K., Sharmaa P.K. Rajesh purohitb, \& Ranab, R.S. A Review on Electromagnetic Forming Process. Procedia Materials Science. 2014. Vol. 6. Pp. 520-527. DOI: https://doi.org/10.1016/j.mspro.2014.07.066.

6. Batygin Y., Barbashova M., Sabokar O. Electromagnetic Metal Forming for Advanced Processing Technologies. Springer, Cham., 2018. DOI: https://doi.org/10.1007/978-3-319-74570-1.

7. Rudnev V., Loveless D., Cook R., Black M. Handbook of induction heating. London: Taylor \& Francis Ltd, 2017. 772 p. DOI: https://doi.org/10.1201/9781315117485.

8. Lucía O., Maussion P., Dede E.J., Burdío J.M. Induction Heating Technology and Its Applications: Past Developments, Current Technology, and Future Challenges. IEEE Transactions on Industrial Electronics. 2014. Vol. 61. Issue 5. Pp. 2509 - 2520. DOI: https://doi.org/10.1109/TIE.2013.2281162.

9. Acero J., Alonso R., Burdio J.M., Barragan L.A., Puyal D. Analytical equivalent impedance for a planar induction heating system. IEEE Transaction on Magnetics. 2006. Vol. 42. No 1. Pp. 84-86. DOI: https://doi.org/10.1109/TMAG.2005.854443.

10. Vasetsky Y.M., Kondratenko I.P., Rashchepkin A.P., Mazurenko I.L. Electromagnetic interactions between current contours and conductive medium. Kyiv: Pro Format, 2019. 221 p. (Rus).

11. Rytov S.M., Calculation of skin effect by perturbation method. Journal of Experimental and Theoretical Physics. 1940. Vol. 10. Issue 2. Pp. 180-190. (Rus).

12. Yuferev S., Ida N. Surface Impedance Boundary Conditions: A Comprehensive Approach. CRC Press, 2018. 412 p. DOI: https://doi.org/10.1201/9781315219929.

13. Leontovich M.A. On the Approximate Boundary Conditions for Electromagnetic Field on the Surface of Highly Conducting Bodies. Propagation of electromagnetic waves. Moscow. USSR Academy of Sciences Publ., 1948. Pp. 5-20 (Rus).

14. Landau L.D., Lifshitz E.M. Electrodynamics of Continuous Media. Elsevier Ltd, 1984. 475 p. DOI: https://doi.org/10.1016/B978-0-08-030275-1.50024-2.

15. Berdnik S.L., Penkin D.Y., Katrich V.A., Penkin Yu.M., Nesterenko M.V. Using the concept of surface impedance in problems of electrodynamics (75 years later). Radio Physics and Radio Astronomy. 2014. Vol. 19. No 1. Pp. $57-$ 80. DOI: https://doi.org/10.15407/rpra19.01.057. 
16. Liu X., Yang F., Li M., Xu S. Generalized Boundary Conditions in Surface Electromagnetics: Fundamental Theorems and Surface Characterizations. Applied Sciences. 2019. Vol. 9. Issue 9. Pp. 1891- 1918. DOI: https://doi.org/10.3390/app9091891.

17. Vasetsky Yu.M., Dziuba K.K. An analytical calculation method of quasi-stationary three-dimensional electromagnetic field created by the arbitrary current contour that located near conducting body. Tekhnichna Elektrodynamika. 2017. No 5. Pp. 7-17. (Rus). DOI: https://doi.org/10.15407/techned2017.05.007.

18. Vasetsky Yu.M., Dziuba K.K. Three-dimensional quasi-stationary electromagnetic field generated by arbitrary current contour near conducting body. Tekhnichna Elektrodynamika. 2018. No 1. Pp. 3-12. DOI: https://doi.org/10.15407/techned2018.01.003.

19. Dzjuba K., Mazurenko I. and Vasetsky Y. An assessment of accuracy of approximate mathematical model of pulse electromagnetic field of current flowing near conductive body. 16th International Conference on Computational Problems of Electrical Engineering (CPEE). Lviv, Ukraine. 2015. Pp. 36-38. DOI: https://doi.org/10.1109/CPEE.2015.7333331.

20. Vasetsky Yu. Nonuniform electromagnetic field at the interface between dielectric and conducting media. Progress in Electromagnetics Research Letters. 2020. Vol. 92. Pp. 101-107. DOI: https://doi.org/10.2528/PIERL20050802.

21. Vasetsky Yu.M. Penetration of non-uniform electromagnetic field into conducting body. Electrical Engineering \& Electromechanics. 2021. No 2. Pp. 43-53. DOI: https://doi.org/10.20998/2074-272X.2021.2.07.

22. Vasetsky Yu.M. Three-dimensional quasi-stationary electromagnetic field of the current near conducting body. Kyiv: Pro Format, 2019. 212 p. (Rus).

23. Zommerfeld A. Elektrodynamics. Moskva: Izdatelstvo Inostrannoy literatury, 1958. 501 p. (Rus.)

24. Tozoni O.V. Method of secondary sources in the electrical engineer. Moskva: Energiya, 1975. 296 p. (Rus.)

25. Nayfeh A.H.. Introduction to Perturbation Techniques. A Wiley-VCH, 1993. 536 p.

26. Smirnov V.I. Higher Mathematics Course. Vol. 3. Part 2. Moskva: Nauka, 1974. 672 p. (Rus).

27. Korn G, Korn T. Mathematical Handbook for Scientists and Engineers: Definitions, Theorems, and Formulas for Reference and Review. Dover Publications, 2000. 1152 p.

28. Polivanov K.M. Theoretical bases of electrical engineers. Vol. 1. Linear electrical circuits with lumped parameters. Moskva-Leningrad: Energiia, 1965. 360 p. (Rus)

29. Knoepfel H. Pulsed High Magnetic Fields. Canada: John Wiley \& Sons, Limited, 1997. 372 p.

\section{ТОЧНИЙ АНАЛІТИЧНИЙ І НАБЛИЖЕНИЙ АСИМПТОТИЧНИЙ МЕТОДИ РОЗРАХУНКУ ТРИВИМІРНОГО ЕЛЕКТРОМАГНІТНОГО ПОЛЯ ПОБЛИЗУ ЕЛЕКТРОПРОВІДНОГО ТІЛА 3 ПЛОСКОЮ ПОВЕРХНЕЮ}

Ю.М. Васецький, докт. техн. наук

Інститут електродинаміки НАН України, пр. Перемоги, 56, Київ, 03057, Україна

e-mail: yuriy.vasetsky@gmail.com

Розглядається аналітичний розв'язок задачі розрахунку тривимірного квазістачіонарного електромагнітного поля струму, щзо протікає поблизу електропровідного тіла з плоскою поверхнею. Представлено точний $i$ наближений розв'язок задачі для синусоїдального та імпульсного полів. Точний розв'язок не має обмежень по конфігурачії зовнішнього поля, електрофізичним властивостям середовища і частоті поля. Наближений розв'язок заснований на розкладанні виразів в асимптотичні ряди і має обмеження: для синусоїдального поля частотами вище нижньої межі; для імпульсного поля - початковим проміжком часу дї імпульсу струму. На основі порівняння результатів точного і наближеного розрахунків для неоднорідного синусоїдального поля біля межі поділу середовищ визначено допустиме значення введеного малого параметра. Для імпульсного поля обтрунтовано запропонований вибір обмеженого проміжку часу розрахунку з використанням асимптотичного методу. Бібл. 29, рис. 7.

Ключові слова: тривимірне електромагнітне поле, точний аналітичний метод, розкладання в асимптотичний ряд, скін-ефект, синусоїдальне та імпульсне поля.

Надійшла 27.05.2021 